\title{
Special Relativity, Coexistence and Temporal Parts: A Reply to Gilmore
}

\author{
Yuri Balashov \\ Department of Philosophy, University of Georgia, Athens, GA 30602, USA \\ yuri@uga.edu
}

[July 29, 2004]

[Forthcoming in Philosophical Studies]

\begin{abstract}
In two earlier works (Balashov, 2000a and 2000b), I have argued that considerations based on special relativity and the notion of coexistence favor the perdurance view of persistence over its endurance rival. Cody Gilmore (2002) has subjected my argument to an insightful three-fold critique. In the first part of this paper I respond briefly to Gilmore's first two objections. I then grant his observation that anyone who can resist the first objection is liable to succumb to the third one. This, however, opens a way to other closely related relativistic arguments against endurantism that are immune to all three objections and, in addition, throw new light on a number of important issues in the ontology of persistence. I develop two such novel arguments in the second half of the paper.
\end{abstract}

\section{Introduction}

The impact of relativity theory on the metaphysical debate about persistence over time has drawn well-deserved attention lately. ${ }^{1}$ In two earlier works (Balashov, 2000a and 2000b), I have argued that considerations based on special relativity (SR) and the notion of coexistence favor the perdurance view of persistence over its endurance rival. In a recent paper, Cody Gilmore (2002) has offered three objections to my argument. I begin by responding briefly to the first two objections. I then concede Gilmore's point that resisting the first objection significantly weakens one's position vis-à-vis the third one. At that juncture, however, the dialectic of the dispute suggests two stronger relativistic

${ }^{1}$ See Rea, 1998; Balashov, 1999, 2000a, 2000b, 2000c, 2002, 2003; Sider, 2001, Section 4.4; Hudson, 2002, 2003; Hales and Johnson, 2003. 
Yuri Balashov

arguments favoring perdurantism over endurantism, which prevail over the disjunction of all three objections.

Since Gilmore's reconstruction of my original argument is remarkably clear and serves so well to highlight the points of his criticism, I take the liberty to use it in rehearsing the argument below. Space constraints prevent me from addressing all the points he has raised (this will have to await a more comprehensive treatment). Accordingly, I focus on the tension between two separate components of my view, which Gilmore has brought to light, and suggest a way of overcoming it. This serves to strengthen my case and advance the discussion of rival theories of persistence in the context of SR.

\section{Background and Preliminaries}

1.1. Doing it the hard way. This debate presupposes eternalism - the view that all spacetime regions and their occupants have the same ontological status - and rejects the link between endurantism and presentism. To show that SR favors perdurantism over endurantism, it is not enough to show that SR rules out presentism; one has to produce an independent argument.

1.2. Idealization. We assume that enduring and perduring material objects do not have spatial extension. Nothing of substance turns on this idealization.

1.3. Spatiotemporal locations of persisting objects. A four-dimensional perduring object, on this assumption, exactly occupies a distinct worldline, and a momentary part of such an object occupies a single spacetime point. An enduring object, on the contrary, typically occupies multiple spacetime points at different moments of its career, which could be indexed by the common absolute time in the classical case or by the proper time of the object in question in the relativistic case.

1.4. Coexistence. Both endurantism and perdurantism must incorporate an interesting - neither empty nor universal-notion (or notions) of coexistence. On anyone's view, there must be a sense of the coexistence relation such that I bear this relation to George W. Bush but not to Napoleon. This much I take to be uncontroversial. What is controversial (and Gilmore's paper makes it very clear) is the link between this notion and that of existence. I return to this point in Section 3.

I believe (but Gilmore does not; see Section 2 for his objections to this thesis and my reply) that coexistence of two arbitrary enduring or perduring objects must be grounded in an objective relation between their spatiotemporal locations. Such a relation defines the basic notion of coexistence (BASC), which, in turn, gives rise to a family of derivative notions, some familiar some new. For enduring objects, the basic notion, BASC-E, is a four-place relation involving two objects $\mathrm{E}_{1}$ and $\mathrm{E}_{2}$ and their respective spatiotemporal locations $\mathrm{O}_{1}$ and $\mathrm{O}_{2}$. BASC-E obtains just in case $\mathrm{E}_{1}$ and $\mathrm{E}_{2}$ are wholly 
Relativity, Coexistence and Temporal Parts

present at (wP@) $\mathrm{O}_{1}$ and $\mathrm{O}_{2}$ respectively and the relevant two-place relation $R$ holds between spacetime points $\mathrm{O}_{1}$ and $\mathrm{O}_{2}$ :

$$
B A S C-E\left(\mathrm{E}_{1}, \mathrm{E}_{2}, \mathrm{O}_{1}, \mathrm{O}_{2}\right) \leftrightarrow \mathrm{E}_{1} \mathrm{wP} @ \mathrm{O}_{1} \& \mathrm{E}_{2} \mathrm{wP} @ \mathrm{O}_{2} \& R\left(\mathrm{O}_{1}, \mathrm{O}_{2}\right)
$$

The nature of $R$ is determined by a particular spacetime theory. In the classical framework, this relation is, informally, that of absolute simultaneity and can be defined (informally) as follows:

$$
R^{\mathrm{N}}\left(\mathrm{O}_{1}, \mathrm{O}_{2}\right)=_{\mathrm{df}}(\exists t)\left(\mathrm{O}_{1} \in \mathrm{HPS}^{t} \& \mathrm{O}_{2} \in \mathrm{HPS}^{t}\right)
$$

Here HPS $^{t}$ is the hyperplane of simultaneity corresponding to time $t$ in some inertial frame and the subscript ' $\mathrm{N}$ ' stands for 'neo-Newtonian'. On BASC-E $\mathrm{E}^{\mathrm{N}}$, then, two enduring objects coexist just in case their momentary locations are contemporaneous, or co-present. Such locations can be conveniently individuated by dates or, in some cases, by the ages of the objects in question. The 51-year old president Putin coexists, in the basic sense of BASC-E ${ }^{\mathrm{N}}$, with the 57-year-old president Bush, but not with the 55-yearold Bush. Alternatively (if less elegantly), Putin, when he is wholly present at some moment in 2004, coexists with Bush, when he is wholly present at the same moment, but not when Bush is wholly present at some moment in 2002. Admittedly, BASC-E is a theoretical concept that is at some remove from more intuitive notions. But it enjoys the sort of generality allowing it to serve as a common ground for various such notions. (It is similar, in this respect, to the concept of spatial location, which is normally taken to be fundamental; more empirical notions such as distance, angles in space, and so on can then be defined on its basis.) For example, a more recognizable triadic relation ' $E_{1}$ coexists with $\mathrm{E}_{2}$ at $t$ ' can be defined, classically, as ' $\left(\exists \mathrm{O}_{1}, \mathrm{O}_{2}\right)\left(\mathrm{E}_{1}\right.$ wP@ $\mathrm{O}_{1}$ \& $\mathrm{E}_{2}$ wP@ $\mathrm{O}_{2}$ \& $\left.\mathrm{O}_{1}, \mathrm{O}_{2} \in \mathrm{HPS}^{t}\right)^{\prime}$. Putin and Bush coexist, in this sense, in 1955 but not in 1950. Similarly, the dyadic relation ' $\mathrm{E}_{1}$ coexists with $\mathrm{E}_{2}$ ' obtains between two enduring objects just in case they 'overlap' temporally: $\left(\exists \mathrm{O}_{1}, \mathrm{O}_{2}\right)(\exists t)\left(\mathrm{E}_{1}\right.$ wP@ $\mathrm{O}_{1} \& \mathrm{E}_{2}$ wP@ $\left.\mathrm{O}_{2} \& \mathrm{O}_{1}, \mathrm{O}_{2} \in \mathrm{HPS}^{t}\right)$. Putin coexists, in this sense, with Bush but not with Napoleon. Below I allow myself the liberty to use these different relations of coexistence without explicitly noting their adicity where context makes it clear which notion is at work.

What about perduring objects? The ground-level notion of coexistence appropriate for them is the dyadic relation BASC-P between the locations of their momentary temporal parts:

$$
\text { BASC-P }\left(\mathrm{P}_{1}^{\mathrm{O}_{1}}, \mathrm{P}_{2}{ }^{\mathrm{O}_{2}}\right) \leftrightarrow R\left(\mathrm{O}_{1}, \mathrm{O}_{2}\right)
$$

Here ' $\mathrm{P}_{1}{ }^{\mathrm{O}_{1}}$, and ' $\mathrm{P}_{2}{ }^{\mathrm{O}_{2}}$, denote momentary temporal parts of perduring objects $\mathrm{P}_{1}$ and $\mathrm{P}_{2}$ located at $\mathrm{O}_{1}$ and $\mathrm{O}_{2}$ respectively. But the perdurantist may also be interested in derivative relations of coexistence, such as that between a temporal part of $\mathrm{P}_{1}$ (say, $\mathrm{P}_{1}{ }^{\mathrm{O}_{1}}$ ) 
and $\mathrm{P}_{2}$ taken as a whole, which obtains iff $\mathrm{P}_{2}$ has a temporal part related to $\mathrm{P}_{1}{ }^{\mathrm{O}_{1}}$ by BASC-P, or the relation of temporal 'overlap' holding between two four-dimensional objects just in case they have temporal parts related by BASC-P.

1.5. Coexistence and temporal modification. The endurantist notions of coexistence are temporally qualified in a way the corresponding perdurantist notions are not. In Gilmore's words, "for the endurantist, all coexistence is coexistence at; and for the perdurantist, all coexistence is coexistence simpliciter" (2002, p. 250). Indeed, BASC-E is a tetradic relation involving two objects and two temporal modifiers: spatiotemporal locations or, alternatively, dates, proper times or ages of the objects in question. Thus I at 1961 coexist, in the basic sense, with JFK at 1961. This serves to ground a more familiar sense in which I coexisted with JFK in 1961-when we were both fully present at a certain moment in 1961 - but do so no longer. The perdurantist notions of coexistence originating from BASC-P are, on the contrary, dyadic and free of temporal modification. They are also free of temporal connotations (or so I argue below). It would be entirely inappropriate to say that my 1961 part used to coexist with (the relevant part of) JFK but does so no longer. Nor would it be strictly appropriate to say that my entire fourdimensional worldworm used to do so. More on this below.

1.6. Coexistence in Minkowski spacetime. In classical spacetime coexistence and its derivatives introduced above are based on the notion of co-presence at a single moment of absolute time. This notion is not available in SR. In Balashov, 2000a and $2000 \mathrm{~b}$, I have argued that the best candidate to ground coexistence in Minkowski spacetime is the invariant relation of spacelike separation between momentary locations of enduring objects, or of parts of perduring objects:

$$
R^{\mathrm{M}}\left(\mathrm{O}_{1}, \mathrm{O}_{2}\right)={ }_{\mathrm{df}} \eta\left(\mathrm{O}_{1}, \mathrm{O}_{2}\right)<0 \text {, }
$$

where $\eta \equiv c^{2}\left(t_{2}-t_{1}\right)^{2}-\left(x_{2}-x_{1}\right)^{2}-\left(y_{2}-y_{1}\right)^{2}-\left(z_{2}-z_{1}\right)^{2}$ is the Minkowski interval. Following Gilmore, let me refer to this general proposal as CASS (Coexistence As Spacelike Separation).

CASS emerges as the best candidate for a number of reasons (see Balashov, 2000a, pp. 132-152). Here I wish to note that, once we settle on Objectivity (see below), the range of choices becomes rather limited, for the relevant relation has to be based on the invariant structures of Minkowski spacetime. Spacelike separation then appears to be the most plausible option, which also correctly recovers the classical limit (i.e., the notion of co-presence at a moment of absolute time). But there is a more general worry about spacelike separation as the ground of an interesting notion of coexistence. Given the lack of causal connectability between spacelike separated events, does this relation have any ontological 'oomph'? And what does it have to do with the commonsense notion of coexistence, which appears to be based on the presence of extensive causal transactions between coexisting entities, such as you and me? I acknowledge that CASS is somewhat removed from the everyday notion, but I maintain that it has strong theoretical 


\section{Relativity, Coexistence and Temporal Parts}

credentials. Looking at a distant star and realizing that the light one is now perceiving was emitted thousands of years earlier, it is perfectly reasonable to ask if the star still exists. True, the question has some classical overtones. But clearly, what drives it is a well-placed concern about a relation between causally unconnected items, a relation in the ballpark of CASS. ${ }^{2}$

It is also clear that, under the assumption that causal interactions cannot propagate faster than a certain limit, the issue of causal connectability cuts across the classicalrelativistic distinction. In particular, the items related by the classical limit of CASS are causally disconnected. But such items belong to the common classical 'present'. Indeed, they exhaust what there is for the presentist, who will be happy to add that all such items coexist. This is not to endorse presentism, but only to indicate that causal connectability and coexistence are conceptually distinct and may go their separate ways. coexistence.

CASS gives rise to the following relativistic analogs of the classical accounts of

(CE) Two enduring objects $\mathrm{E}_{1}$ and $\mathrm{E}_{2}$, located at spacetime points $\mathrm{O}_{1}$ and $\mathrm{O}_{2}$ respectively, coexist iff $\mathrm{O}_{1}$ and $\mathrm{O}_{2}$ are spacelike separated: $C E\left(\mathrm{E}_{1}, \mathrm{O}_{1}, \mathrm{E}_{2}, \mathrm{O}_{2}\right)={ }_{\mathrm{df}} B A S C-E^{\mathrm{M}}\left(\mathrm{E}_{1}, \mathrm{E}_{2}, \mathrm{O}_{1}, \mathrm{O}_{2}\right)$.

(CP) Two momentary temporal parts of perduring objects coexist iff their locations are spacelike separated: $C P\left(\mathrm{P}_{1}{ }^{\mathrm{O}_{1}}, \mathrm{P}_{2}{ }^{\mathrm{O}_{2}}\right)={ }_{\mathrm{df}} B A S C-P^{\mathrm{M}}\left(\mathrm{P}_{1}{ }^{\mathrm{O}_{1}}, \mathrm{P}_{2}{ }^{\mathrm{O}_{2}}\right)$.

Just like $B A S C-E^{\mathrm{N}}$, its relativistic counterpart can be used to define derivative notions of coexistence, such as that between $\mathrm{E}_{1}$ located at $\mathrm{O}_{1}$ and $\mathrm{E}_{2}$ taken in abstraction from its spacetime location, as well as the notion of relativistic 'temporal overlap'. Similarly for $B A S C-P^{\mathrm{M}}$.

Before endorsing CASS, I had considered several other candidates and had shown that they fail to meet certain requirements one can impose on the notion of coexistence, the most important of them being Objectivity:

(Objectivity) Given two objects, or their momentary temporal parts, having determinate locations in spacetime, there must be a fact of the matter about their coexistence.

This brings me to the first point of Gilmore's critique.

2 See Sections 3, 6.3, 6.4, and 7 for detailed discussions of the conceptual link between Coexistence and what I call Existence@. 


\section{Gilmore's First Objection}

Gilmore rejects CASS in favor of the following account (2002, p. 254; I have slightly paraphrased Gilmore's original formulation):

(REL) Coexistence is relative to a hyperplane of simultaneity (HPS). For any two enduring or perduring objects and any HPS, the objects in question coexist at this HPS iff their worldlines intersect it.

Gilmore cites a number of reasons to prefer REL to CASS, chief among them being that, unlike CASS, REL makes the relevant notion of coexistence go hand in hand with simultaneity and, in addition, makes it a transitive relation (for a given HPS). Both reasons, in my mind, have a classical origin and lose much of their force in making a transition to something as full of surprises as Minkowski spacetime. I have more to say about transitivity in Sections 6.1 and $7 .^{3}$ But in any event, the alleged advantages of REL are far from being decisive and are outweighed by what I take to be its major weakness, the fact that it violates the Objectivity requirement.

To put the point in a deliberately grotesque form, on REL, there is no fact of the matter as to whether I coexist with Cody Gilmore, and this regardless whether we are enduring or perduring objects. Neither is there a fact of the matter as to whether Cody coexists with any of his dissertation committee members. Indeed, there is no fact of the matter about the coexistence of any two objects, including those whose histories closely overlap. Even a pair of interwoven threads in a tapestry, which come to be and cease to exist together (let us suppose), do not coexist in any objective sense because, on REL, they do not coexist relative to a hyperplane of simultaneity drawn throw the temporal beginning or the end of one of them 'slightly at an angle', so that it does not intersect the worldline, or the worldworm, of the other. I take this result to come very close to a reductio of REL and conclude that CASS, and hence BASC, hold up well in comparison. Coexistence must be objective.

With CASS and BASC thus rehabilitated, I now turn to a more controversial issue that has come to light in Gilmore's critical study.

\section{Coexistence and Existence@}

As noted above, one of the classical endurantist notions of coexistence based on BASCthat between an object wholly present at some point and another object considered in

${ }^{3}$ Cf. also Balashov, 2000a, pp. 140-141. Considerations of space do not allow me to do justice to other astute remarks Gilmore has made about REL versus CASS and to a friendly amendment to CASS he has suggested. 


\section{Relativity, Coexistence and Temporal Parts}

abstraction from any of its locations - underlies the sense in which, at any moment of my life, many enduring objects in the universe can be divided into three classes: those with which, intuitively speaking, I coexist no longer; those with which I still or already coexist; and those with which I do not yet coexist. As I grow older, the membership of these classes changes, thanks to the presence in our world of many enduring objects that come to be and cease to exist. To use my older example (Balashov, 2000b), when I was fully present on 2/6/99, I still coexisted with Jordan's King Hussein (who died the next day) and already coexisted with his son, Crown Prince Hassan. A week later, when I was wholly present on $2 / 13 / 99$, I no longer coexisted with the former, still coexisted with the latter, who then became King Abdullah, and not yet coexisted with Abdullah's grandson.

These determinations can be transferred, unscathed, to Minkowski spacetime, as long as we take care to track momentary spatiotemporal locations of a given enduring object by some useful relativistically invariant parameter, for example, the object's proper time (measuring the age of the object in its rest frame). Of course, we now have to employ the relativistic versions of the endurantist coexistence principles (i.e., $\mathrm{CE}$ and its derivatives).

I argued, next, that in both cases, the classical as well as relativistic, there is an interesting notion of existence going hand in hand with coexistence. For a given enduring object, the changing relations of coexistence it enters into during its life career provide a changing perspective on the rest of the world. When I was fully present on 2/6/99, there was a sense in which King Hussein still existed and Crown Prince Hassan already did. A week later, on the other hand, the former existed no longer. What reasons did I have to assert, on 2/13/99, that King Abdullah (= Crown Prince Hassan), but not the late King Hussein, was still in existence? The reason seems clear: Abdullah, but not Hussein, coexisted with me, when I was wholly present on 2/13/99.

Before we go any farther, we need to make sure that the notion of existence just introduced is sensible. Gilmore suggests, in effect, that it is not (2002, pp. 254-255). And there is no doubt that the notion is controversial. In putting it forward, I am setting myself in opposition to one of the cornerstones of analytic philosophy, the thesis that existence is a univocal concept. On the eternalist view presupposed throughout this discussion, past, present, and future entities are equally in existence. This basic tenseless notion of existence applies to the late King Hussein, and even to the Babylonian king Nebuchadnezzar, no less than to King Abdullah. It would appear that adding that the latter still exists but the former two do so no longer cuts no ontological ice but merely serves to say something about the object's temporal (or spatiotemporal) location.

I am not sure that the boundary between the ontological and the locative can always be clearly drawn. But I hope to provide, by the end of this paper, enough support for the thesis (named by Gilmore the Asymmetry Thesis; see below) that the 'locative' notion of existence-let us call it Existence@, to contrast it with existence simpliciterhas significant implications for endurantism, but not for perdurantism, where its role boils down to representing certain 'perspectival' phenomena in spacetime. This, however, 
requires a lot of work, which still lies ahead. At this point, I am concerned to establish the initial credentials of the notion of Existence@. This, by itself, appears to be in defiance of common wisdom. But I think there are good reasons to be defiant.

Concerning the distinction between existence and Existence@, note, first, that one can draw a parallel distinction between two concepts of coexistence, the narrow one discussed in this paper, according to which I coexist with King Abdullah but not with Nebuchadnezzar, and a broader concept, according to which all inhabitants of spacetime (including me and Nebuchadnezzar) coexist with each other. The narrow concept is nontrivial and informative in a way the second is not. And since existence simpliciter goes hand in hand with the trivial notion of coexistence, it is natural to expect that there should be another notion of existence, to go along with Coexistence in the interesting sense. This other notion is, of course, Existence@.

Second, the distinction between existence simpliciter and coexistence in the trivial sense, on one side, and Coexistence in the interesting sense and Existence@, on the other, is similar to another, and famous, distinction that does a great deal of work in modal realism. As noted by David Lewis, to quantify correctly over possibilia and, in general, to do justice to modal discourse, the modal realist needs two different quantifiers: one ranging over the contents of the entire collection of possible worlds and the other restricted to a particular such world (see Lewis, 1986, pp. 3, 5-7, and elsewhere). Any two objects populating the Lewisian multi-universe coexist in the broad sense, but those belonging to different worlds fail to coexist in the restricted sense. An inhabitant of our world can state, with seriousness, that alien objects do not actually exist, meaning their non-existence in our world. (Is such a statement ontological or 'merely locative'? I suppose this is debatable. But no matter how one reads it, the statement is clearly significant.)

The analogy with the temporal case works as follows. Beginning with the classical context, associate with existence simpliciter the unrestricted quantifier ranging over all enduring objects. Now for each particular $t$ held fixed, introduce a restricted quantifier ranging over enduring objects located at $t$ (i.e., at spacetime points belonging to HPS $^{t}$ ). The meaning of 'Existence@' is then associated with the family of such restricted quantifiers.

Consider two enduring objects $\mathrm{E}_{1}$ and $\mathrm{E}_{2}$ located at $t$. Such ' $t$-mates' coexist both in the trivial and in the interesting sense. Consequently, for $\mathrm{E}_{1}$ at $t, \mathrm{E}_{2}$ coexists with it (hence, exists@) still or already. Suppose another enduring object $\mathrm{E}_{3}$ ends its career at $t^{\prime}$ $<t$. Then although $\mathrm{E}_{1}$ at $t$ and $\mathrm{E}_{3}$ at $t^{\prime}$ coexist in the trivial sense, $\mathrm{E}_{1}$ and $\mathrm{E}_{2}$ fail to be $t$ mates. For $\mathrm{E}_{1}$ at $t, \mathrm{E}_{3}$ no longer coexists with it, hence no longer exists@.

One might object that these locutions betray Existence@ as a relation to time and 'E exists@' as an incomplete expression, and that this is inappropriate to any concept of existence deserving the name. To this, I reply that the concept of existence at a world corresponding to the Lewisian restricted quantifier can similarly be looked upon as involving a relation to some inhabitant of a given world (viz., the world-mate relation) 


\section{Relativity, Coexistence and Temporal Parts}

and the corresponding expression 'E exists' (meaning its existence in that world) as an incomplete expression. If that is unproblematic (which I think it is), then I don't see why the relational (in a similar sense) nature of Existence@ should be problematic. In essence, Existence@ behaves a lot like the indexical notion of actuality does in modal realism. Any object that is a world-mate of a given object is actual. Similarly, for any enduring object wholly present at $t$, another enduring object exists@ just in case it is also wholly present at $t$. Statements of actuality in modal realism go hand in hand with statements of restricted coexistence, except that the former suppress mention of the object with respect to which actuality is covertly relative, in virtue of the world-mate relation. Similarly, statements of Existence@ go hand in hand with statements of Coexistence, except that the former suppress mention of the pair consisting of an object and a time (or a spacetime point) with respect to which Existence@ is covertly relative, in virtue of the $t$-mate relation.

The relativistic generalization of Existence@ requires replacing the relation of belonging to the same (moment of absolute) time with the relation of spacelike separation. If I am wholly present here and now (at $\mathrm{O}$ ), any other enduring object exists@ just in case it coexists with me now (i.e., is wholly present at $\mathrm{O}^{*}$ spacelike separated from O). Moreover, it is appropriate to qualify the Existence@ of such objects with temporal modifiers still, already, not yet, and the like. It is especially appropriate when the objects in question come to be and cease to exist 'during' my own life career.

This gives rise to the following situation represented in Figure 1. (Here I am using my example from Balashov, 2000b, p. S560.) Suppose I am wholly present at a certain moment of my proper time somewhere on Betelgeuse. Then I still coexist with King Hussein and I already coexist with Hussein's great-grandson. Moreover, in view of the connection between Coexistence and Existence@, King Hussein still exists@, and his great-grandson already does. In Gilmore's apt expression (2002, p. 245), they are both 'temporally here' for me now. But this, I have argued, is unacceptable. There is no temporally-laden sense in which King Hussein and his great-grandson can be in existence or 'temporally here' together: the former's end lies in the absolute past of the latter's beginning. Since the endurantist is committed to this temporally-laden 'togetherness' claim in the relativistic context, endurantism is inferior to perdurantism.

Figure 1.

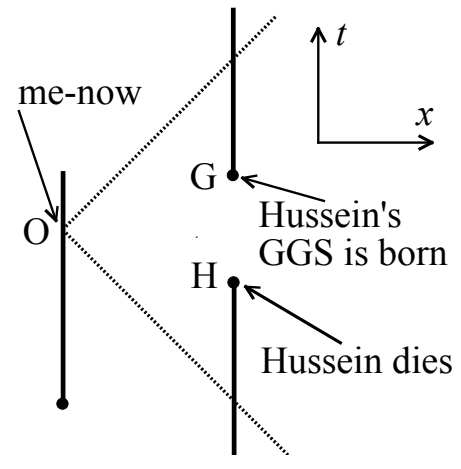


This is surely a lot going on here. One way to unpack it, which was suggested by Gilmore, is to identify two separate claims that come together to produce the above conclusion, the Asymmetry Thesis and the Absurdity Thesis. According to the former, the endurantist is, but the perdurantist is not committed to the temporally-laden (and hence, potentially threatening) reading of the togetherness claim. According to the Absurdity Thesis, this commitment is harmful to endurantism. Gilmore objects to both theses. In the next section I respond to his objection to the Asymmetry Thesis.

\section{The Asymmetry Thesis: Gilmore's Second Objection}

What makes King Hussein and his great-grandson 'temporally here' for me now-when I am located at $\mathrm{O}$ - is that, when I am so located, I coexist with both of them in one of the derivative senses of the basic endurantist coexistence relation $B A S C-E^{\mathrm{M}}$. The principle governing this derivative relation (call it Coexistence*) is as follows:

(CE*) An enduring object $\mathrm{E}_{1}$ wholly present at $\mathrm{O}_{1}$ coexists* with another enduring object $\mathrm{E}_{2}$ considered in abstraction from its location just in case there is a spacetime point $\mathrm{O}_{2}$ such that $\mathrm{E}_{2}$ is wholly present at $\mathrm{O}_{2}$ and $\mathrm{O}_{2}$ is spacelike separated from $\mathrm{O}_{1}$.

But the corresponding perdurantist principle is easily available too:

(CP*) The $\mathrm{O}_{1}$-part of perduring object $\mathrm{P}_{1}$ coexists* with another perduring object $\mathrm{P}_{2}$ iff $\mathrm{P}_{2}$ has a part located at a spacetime point $\mathrm{O}_{2}$ that is spacelike separated from $\mathrm{O}_{1}$.

This suggests that the perdurantist may also be entitled to an interesting notion of existence-call it Existence ${ }^{\mathrm{P}} @$ similar to the endurantist notion of Existence@. In particular, the perdurantist may want to say that, for any momentary part of perduring object $\mathrm{P}$ located at $\mathrm{O}$, another perduring object exists ${ }^{\mathrm{P}} @$ just in case it has a part located at a spacetime point that is spacelike separated from $O$. And this implies that in scenarios such as one involving King Hussein and his great-grandson, the perdurantist is committed to an analog of the 'togetherness claim'. Suppose I am a perduring object and one of my parts is located at a certain moment $t$ of my proper time somewhere on Betelgeuse. Call this part Yuripart. According to CP*, Yuripart coexists* with King Hussein and it also coexist* with Hussein's great-grandson. Moreover, in view of the connection between Coexistence* and Existence ${ }^{\mathrm{P}} @$, King Hussein exists ${ }^{\mathrm{P}} @$ and so does his great-grandson. They are both 'temporally here' for Yuripart. It would seem that if the togetherness claim is unacceptable for the endurantist, then the perdurantist is no better off. 


\section{Relativity, Coexistence and Temporal Parts}

My reasons for thinking otherwise are based on two distinctive features of the endurantist ontology. The first such feature is that (a) an enduring object is wholly present at each moment of its proper time. (a) is definitive of the very concept of endurantism. My full presence at a certain place and time is, according to this concept, the most important fact about my existence and the focal point of my connection with the rest of the universe. The second distinctive feature (b) of enduring objects is that they change their location with their proper time, and this induces change in relations of such objects to their environment. As I become older, the membership of the class of objects with which I coexist*-hence of those that exist@-undergoes change. This legitimizes ascribing to my coexistence* with them, and hence to their existence@, temporally-laden determinations, such as still, already and the like. This, in turn, leads the endurantist to the togetherness claim, which, I have contended, is unacceptable.

There is no place for anything like (a) and (b) in the perdurantist ontology. If I am a perduring object, I am never fully present at any one place and time. At any such location, I am present only partially, and being only partially present there does not entitle me to temporally-laden determinations, as regards the existence@ of other objects and their coexistence* with me, that being fully present does. Moreover, there is no sense in which perduring entities or their parts change their location in spacetime, no strict sense in which they age, and thus no robust sense in which their coexistence* with other objects (and hence those objects' existence $($ ) can be ascribed changing temporallyladen qualifications (still, already, no longer, etc.). The perdurantist relation Coexistence* holds between, and the property Existence ${ }^{\mathrm{P}}$ @ is possessed by, entities that are atemporally confined to their locations in spacetime. It is therefore inappropriate to say that my 2/6/99 temporal part 'used' to coexist with King Hussein but does so 'no longer'. It is, arguably, also inappropriate to say that my entire four-dimensional worldworm used to coexist with him on 2/6/99 (more on this below).

Absent such temporally-laden connotations, the perdurantist notions of Coexistence* and Existence $^{\mathrm{P}}$ @ give rise, in the case of Hussein and his great-grandson, to the following innocuous situation. My part referred to above as Yuripart, Hussein's last part, and his great-grandson's first part are all confined to their locations in Minkowski spacetime, and the fact that Yuripart coexists* with both is simply a further tenseless fact about us and our parts. The perdurantist version of the togetherness claim is therefore entirely harmless. This establishes the Asymmetry Thesis.

To recap, being wholly present at a spacetime point determines an enduring object's changing relations to the rest of the universe in a way being only partially present at such a point does not for a perduring object. But Gilmore has pressed the charge that although one cannot expect this service from a total four-dimensional perduring object, it could still be performed by its momentary temporal part. Such a part is wholly present at a single spacetime point. Doesn't this allow one to associate with it a definite perspective on the rest of the world, thus restoring parity with endurantism? Moreover, isn't there a sense in which this perspective is also temporally-laden. As 
Gilmore notes, "Insofar as perdurantism can be taken seriously, it must be consistent with ... [the] undeniable fact that there is at least some weak sense in which George W. Bush is still or already in existence for me at the current point on my worldline" (2002, p. 252).

But is this weak sense strong enough to yield a togetherness claim as offending as the corresponding endurantist claim is? I don't think so. The perdurantist claim is grounded in the perspective of a momentary temporal part of a perduring object, such as Yuripart. But in the perdurantist ontology, Yuripart is not me but only a short stage of me. So what is wholly present at a spacetime point - and what has a specific relation to the rest of the world - is not an ordinary object, Yuri, but a less familiar entity, Yuripart. In contrast, what is the focal point of such a relation in the case of endurantism is the ordinary enduring person in its entirety. The contrast is quintessential to the whole issue between endurantism and perdurantism. I submit that it cuts deep enough to render the perdurantist version of the togetherness claim ontologically innocent. The "weak sense," which is at work in Gilmore's objection, is the vicarious sense in which the properties of temporal parts of perduring objects can be attributed to four-dimensional wholes. ${ }^{4}$ Strictly speaking, Yuripart represents $m y$ perspective on the world no more than Yuripart $_{1}$, Yuripart 2 , and countless other temporal parts of me do and thus cannot bear the weight of temporally-laden determinations, such as still and already, pertaining to the existence of other objects. Such an object may be already in existence for Yuripart ${ }_{1}$ but not yet for Yuripart 7 . And since neither Yuripart 1 , nor Yuripart ${ }_{7}$, nor any other temporal part of me represents $m y$ perspective par excellence, it is unclear what we ought to say about the existence (i.e., existence ${ }^{\mathrm{P}} @$ ) of the object in question. On the other hand, if I am an enduring object wholly present at $t$, then my relation to other objects, associated with my temporary location, is determinate and non-vicariously mine.

This underscores the extent to which the ontology of perdurantism is revisionary. It denies that objects have temporary properties and locations in anything stronger than a vicarious sense. Indeed, it is this denial that allows perdurantism to avoid the problem of coincident objects ("temporal overlap is not coincidence"), which constitutes one of the strongest reasons in its favor (see Heller, 2002 for a recent discussion). As usual,

\footnotetext{
${ }^{4}$ In this respect, the stage theory (Sider, 2001, Section 5.8; Hawley, 2001), historically the second variety of four-dimensionalism, which takes ordinary objects to be (rather than to have) momentary stages, does better than the more traditional perdurantism, or the worm theory. On the stage theory, temporary properties and momentary locations are possessed, in the strict sense, by ordinary objects rather than by their parts. My argument (in Balashov, 2000a, 2000b) would, therefore, be ineffective as a defense of the stage-theoretic version of four-dimensionalism against endurantism. But the stage theory has its share of problems; see, e.g., Haslanger, 2003, Section 6, and Balashov, 2002, where I discuss and defend other relativistic arguments, this time favoring the worm theory over the stage theory. Importantly, they ride on a feature that makes the stage theory closer to endurantism than to perdurantism, viz., the idea that persisting objects themselves (not their parts) are wholly present at all moments at which they exist. This proximity makes endurantism vulnerable to versions of the relativistic arguments against the stage theory developed in Balashov, 2002, and serves as the basis of later sections of the present paper.
} 
strength comes with a price tag: revisionism. But perdurantism is no more revisionary than its natural partner, the B-theory of time, which has to be decidedly deflationary about many common-sense notions, such as change, the 'passage' of time, and our different attitudes toward the past and the future. Evaluating a particular ontology requires taking into account the entire body of evidence for and against it, experiential as well as theoretical. Revisionary though it is, perdurantism is a major contender in the debate about persistence.

This concludes my defense of the Asymmetry Thesis. (There is more to say in response to Gilmore's critique of it, but I have to limit myself to the bare essentials here.) In conjunction with CASS, it commits the endurantist to the temporally-laden version of the togetherness claim, which was briefly described above. To repeat, if I am wholly present at a certain far-away point, I coexist* with King Hussein (who is no longer alive for us) and also coexist* with his great-grandson (who is not yet born). This authorizes me to say that they both exist@-both are 'temporally here' for me. And this, I have argued, is unacceptable. Hussein and his great-grandson cannot be in existence together in any temporally-laden sense. Claims of this sort are absurd. But Gilmore has argued that they are not.

\section{The Absurdity Thesis: Gilmore's Third Objection}

More precisely, Gilmore has argued that someone who subscribes to CASS (Coexistence As Spacelike Separation) should not be offended by the temporally-laden version of the togetherness claim. CASS by itself involves such a radical departure from our naïve intuitive ideas about coexistence that making a further step by accepting the consequences of the togetherness claim should not be especially troubling. Thus Gilmore:

If I am willing to broaden my ideas about coexistence so as to allow for the possibility that I now coexist with both of two things that never coexist with each other, then I should also be willing to broaden my ideas associated with the phrase "are still or already in existence for me": I should then be willing to broaden these latter ideas so as to allow for the possibility that there is at least some weak sense in which the given phrase can apply to both of two things that never coexist with each other. (Gilmore, 2002, p. 246)

Is there room for disagreement here? I suppose there is. The whole issue boils down, at this point, to the question of how to extend our ordinary notions into the relativistic domain: what can be sacrificed and what has to be retained at all costs. And since we have already left behind many ordinary beliefs (e.g., those about absolute simultaneity and universal time), we are swimming in uncharted waters. Consequently, there may be no shared standards that could resolve the issue of how to extrapolate old concepts into the new domain to everyone's satisfaction. One might still insist that the endurantist 
should accept CASS, simply because it is the only plausible extension of the interesting sense of coexistence into the relativistic domain, and, at the same time, reject the temporally-laden togetherness claim. But instead of pursuing this line, let me concede that the case is not conclusive. Having defended CASS and the Asymmetry Thesis against Gilmore's first two objections, I am inclined to agree with him that endorsing CASS takes the sting from the endurantist version of the togetherness claim.

But the foregoing analysis suggests a way of reinforcing the case. Building on the results established so far, I develop, in the remainder of this paper, two other arguments of the same broad variety (i.e., also centered on the notion of coexistence), which show that the togetherness claim, even if relatively innocent by itself, is just one species of a family of progressively more harmful consequences of the general idea that coexistence in Minkowski spacetime hinges on spacelike separation, and that the pressure on the endurantist to reject those other consequences is greater than the pressure, defied by Gilmore (and, I suppose, by others), to reject the simple togetherness claim. ${ }^{5}$

\section{Coexistence As Sharing a Hyperplane of Simultaneity}

6.1. From CASS to CASH. Constructing the new arguments requires a generalization of CASS and of the ensuing principles of coexistence. All these principles define coexistence as a relation holding between a pair of entities located at particular spacetime points. As a result, the principles become rather restrictive. The potentially offending endurantist version of the togetherness claim turns almost entirely on this overly restrictive character of $\mathrm{CE}$ and $\mathrm{CE}^{*}$. Indeed, the situation it describes involves an enduring object $\mathrm{E}$ wholly present at spacetime point $\mathrm{O}$ and two other enduring objects $\mathrm{E}_{1}$ and $\mathrm{E}_{2}$, considered in abstraction from their locations, such that $\mathrm{E}$ at $\mathrm{O}$ coexists* with $\mathrm{E}_{1}$, and it also coexists* with $\mathrm{E}_{2}$. This entails that $\mathrm{E}_{1}$ and $\mathrm{E}_{2}$ are both in existence@ (for $\mathrm{E}$ at O). But $E_{1}$ does not coexist with $E_{2}$ in any of the senses allowed by the endurantist principles of coexistence. As we have learned by now, this may or may not be 'absurd'. I want to suggest that whatever trouble there may be in this situation, it should be blamed primarily on the restricted adicity of the coexistence relations defined by $\mathrm{CE}$ and $\mathrm{CE}^{*}$. Given that spacelike separation is not transitive and that coexistence holds between pairs of objects, should someone who is committed to CASS be surprised to discover that in some cases, $\mathrm{E}$ at $\mathrm{O}$ coexists* with $\mathrm{E}_{1}$ and also coexists* with $\mathrm{E}_{2}$, but $\mathrm{E}_{1}$ does not coexist with $\mathrm{E}_{2}$ ? Not necessarily. After all, from the fact that $\mathrm{E}$ at $\mathrm{O}$ coexists* with $\mathrm{E}_{1}$ and $\mathrm{E}$ at $\mathrm{O}$ coexists* with $\mathrm{E}_{2}$, it does not even follow that $\mathrm{E}$ at $\mathrm{O}$ coexists* with $E_{1}$ and $E_{2}$. True,

\footnotetext{
${ }^{5}$ As already noted, the arguments developed below are similar to the earlier relativistic arguments defending the worm theory against the stage theory (Balashov, 2002), despite the difference in context. Below I take the liberty to use, with appropriate modifications, some considerations and examples from my earlier work.
} 


\section{Relativity, Coexistence and Temporal Parts}

the latter can be said to be both in existence (i.e., in existence@) for E at O-but, one wants to add, "not in the same sense." Their existence@ has different indexical modifiers. $\mathrm{E}$ at $\mathrm{O}$ shares a certain hyperplane of simultaneity (HPS) with $\mathrm{E}_{1}$ (when the latter is wholly present at a suitable spacetime point), and it shares another HPS with $\mathrm{E}_{2}$. But there is no single HPS that they all share. ${ }^{6}$ If there were such a common HPS then the situation that led to the allegedly offending togetherness claim would never arise in the first place and, hence, the endurantist would have nothing to worry about.

These observations suggest that, given non-transitivity of the grounding relation of spacelike separation, it may be unfair to start by saddling the endurantist with the dyadic relation of coexistence 7 and then go on to blame her, in essence, for the nontransitive character of this relation. It is also unclear whether grounding the interesting notion of coexistence (i.e., one that is neither empty nor universal) in a relation of fixed adicity is, in general, the best way to treat this notion. It seems reasonable to allow objects to coexist with each other, not merely pairwise, but en masse.

These considerations invite promoting the dyadic relation of coexistence to a multigrade relation. And for anyone who accepts CASS, the natural way to do it is to generalize the relation of spacelike separation between momentary spacetime locations of two entities to the $n$-place relation of belonging to a single HPS. In the hands of the endurantist, the basic idea of Coexistence As Sharing a Hyperplane (of Simultaneity) $(\mathrm{CASH})$ gives rise to the following generalizations of $\mathrm{CE}$ and $\mathrm{CE}^{*}$ (the corresponding perdurantist generalizations of $\mathrm{CP}$ and $\mathrm{CP}^{*}$ are also easily available):

$(\mathrm{CE}-\mathrm{CASH})$ Enduring objects $\mathrm{E}_{1}, \ldots \mathrm{E}_{n}$ located at spacetime points $\mathrm{O}_{1}, \ldots \mathrm{O}_{n}$ respectively coexist iff $\mathrm{O}_{1}, \ldots \mathrm{O}_{n}$ belong to a common HPS.

(CE*-CASH) Enduring object $\mathrm{E}$ wholly present at $\mathrm{O}$ coexists* with enduring objects $\mathrm{E}_{1}, \ldots \mathrm{E}_{n}$, considered in abstraction from their locations, just in case there are spacetime points $\mathrm{O}_{1}, \ldots \mathrm{O}_{n}$ such that $\mathrm{E}_{1}, \ldots \mathrm{E}_{n}$ are wholly present at $\mathrm{O}_{1}, \ldots \mathrm{O}_{n}$ respectively and $\mathrm{O}, \mathrm{O}_{1}, \ldots \mathrm{O}_{n}$ belong to a common HPS.

6.2. CASH versus CASS. For two objects, CASH simply reduces to CASS. But in general, $\mathrm{CASH}$ is an improvement on CASS. It allows one to do justice to the idea that many objects - all the pieces of furniture in my office, all the people currently alive, all the planets in the Solar system, and all the StarTrek characters - can bear a single relation of coexistence to each other. It is true that if $n$ momentary spacetime locations (of enduring objects or of temporal parts of perduring objects) satisfy $\mathrm{CASH}$, then taken

${ }^{6}$ Cf. Gilmore (2002, pp. 258f) who makes essentially the same point, but in a different context, that of defending REL over CASS in his first objection.

${ }^{7}$ Dyadic, in the sense that it relates $\mathrm{E}_{1}$-at- $\mathrm{O}_{1}$ with $\mathrm{E}_{2}$-at- $\mathrm{O}_{2}$. In another sense, noted in Section 1 , this relation (i.e., BASC) is tetradic. 
pairwise, they also satisfy CASS. But the contrary does not hold. In general, for $n>4$, if spacetime points $\mathrm{O}_{1}, \ldots \mathrm{O}_{n}$ are pairwise spacelike separated, it does not follow that $\mathrm{O}_{1}, \ldots$ $\mathrm{O}_{n}$ belong to a common HPS. In this respect, CASH is more comprehensive than CASS. It grants coexistence en masse to a family of objects that, intuitively speaking, coexist all together and not just pairwise.

CASH also eliminates the injustice, which may (as I have conceded) have been done to the endurantist by the Absurdity Thesis, and, hence, it eliminates the reason for Gilmore's objection to this thesis, by precluding the 'absurd' situations from arising in the first place. If I am wholly present at some moment of my proper time on Betelgeuse, then I coexist* with King Hussein and I also coexist* with his great-grandson in the sense of $\mathrm{CE}^{*}$ but not in the sense of $\mathrm{CE}^{*}-\mathrm{CASH}$. And this makes it entirely perspicuous that broadening our ideas about coexistence, in making a transition to the relativistic domain, must go hand in hand, as Gilmore has suggested (2002, p. 246), with broadening our ideas about the use of temporal determinations such as still and already. On CE*-CASH, it is simply not the case that I still coexist with King Hussein and already coexist with his great-grandson. But this is so because I do not coexist* with them at all: we do not share a common HPS. This blocks the next move, the ascription of the temporally-laden sense of existence@ to both of them, at an early stage.

The classical limit of CASH is clearly the old classical notion of existing at the same moment of absolute time. This is also true of CASS. But in the case of CASH, recovering the intuitively correct classical limit is especially valuable, since CASH is broader in scope and thus comes closer to the common notion of coexistence as a multigrade relation, even if it becomes, as a result, less permissive in granting mutual coexistence to a collection of objects.

Our primary interest, however, lies in those features of the relativistic coexistence relations stemming from CASH in which they deviate from their classical counterparts. Before turning to them, let us go back to the important notion of existence@ and introduce its generalized version. It will be convenient to start with the classical (nonrelativistic) context. This may also be a good occasion to switch to different examples.

6.3. Existence@, coexistence, t-mates, and temporal worlds in classical spacetime. There is a familiar sense in which the enduring Descartes, Galileo, Kepler, and Brahe coexisted in 1600, but not in 1620 (Figure 2). The locations of these persons at some point in 1600 belong to the same moment of absolute time (or to a common absolute hyperplane of simultaneity). This is not true of any moment in 1620, because (sadly) Brahe has no location at any moment in 1620. Let us call items sharing a common moment $t$ of absolute time $t$-mates. For the endurantist, such items are enduring objects wholly present at $t$. For the perdurantist, they are momentary $t$-parts of perduring objects.

The enduring Descartes, Galileo, Kepler, and Brahe are 1600-mates but not 1620mates. In the classical context, entities that are $t$-mates coexist (en masse) in the interesting sense and exist $@$ in the sense associated with the restricted quantifier ranging 
Relativity, Coexistence and Temporal Parts

over items wholly present at $t$ (see Section 3). On the other hand, all objects whatsoever - past, present, and future - coexist in the trivial sense, and, of course, all such objects exist (simpliciter). Galileo, Kepler, and Brahe all exist@ for Descartes at 1600, but not for Descartes at 1620. As already noted, they coexist, in the interesting sense, in 1600 , but not in 1620 . On the other hand, all these persons also coexist with each other in the trivial sense, as populating the same four-dimensional spacetime manifold, and, of course, they coexist, in that sense, with everything else that ever existed, exists or will exist in the entire history of the universe.

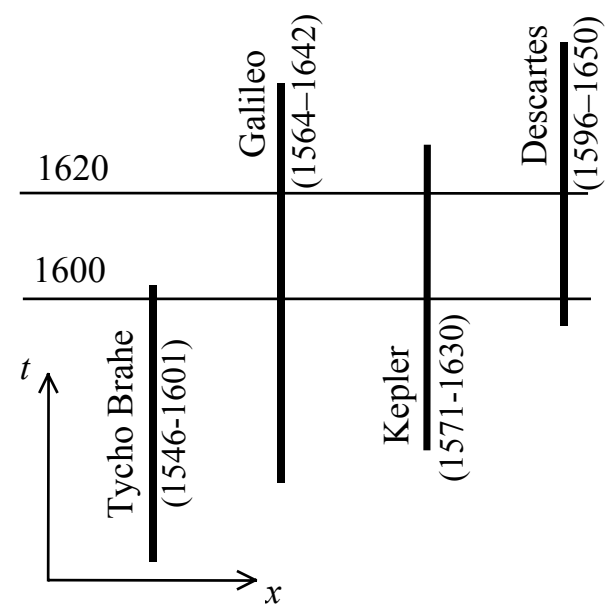

Figure 2.

Just like their dyadic predecessors, the endurantist notions of coexistence and Existence@ are temporally-laden. It makes sense to say that, in 1600, Galileo still coexisted with Brahe and already coexisted with Descartes and Kepler (Figure 2). Galileo continued to coexist with Descartes and Kepler in 1620, but no longer with Brahe. As Galileo grew older, his coexistence relations with other enduring objects in the universe underwent change and, with them, his perspective on their existence. It is perfectly reasonable to say that, for Galileo in 1600, Descartes, Kepler, and Brahe were still in existence (i.e., in existence@). This is no longer the case for Galileo in 1620.

Besides the trivial sense, in which all enduring objects share a single fourdimensional spacetime world, there is, then, an interesting sense in which they can share or fail to share 'temporal worlds' at certain moments of their individual time (which coincides with the common absolute time in the pre-relativistic setting), just as there is an interesting sense in which Lewisian objects may or may not be world-mates, despite the fact that there is a broader sense in which they all coexist by sharing the entire Lewisian pluriverse. The analogy should by now be clear.

There is, however, a notable disanalogy between the worlds of modal realism and temporal worlds. No two Lewisian possible worlds overlap, because no object can exist 
in more than one such world. But if endurantism is true, then distinct temporal worlds (i.e., the occupants of distinct absolute hyperplanes of simultaneity cutting across the entire universe) may contain the same enduring objects. Thus the 1600 -world and the 1620-world both contain the self-same Descartes, Galileo, Kepler and many other enduring objects populating both worlds. This dissimilarity does not preclude one from making a distinction between the two senses of coexistence and, hence, of existence (i.e., the trivial and the interesting). In fact, it underscores the important respect in which Descartes' temporary relations to other objects change with his age: it is the very same Descartes who, first, shares a temporal world with Brahe and, then, fails to share such a world with him.

On the other hand, if perdurantism is true, then distinct classical temporal worlds do not overlap. In this respect, the classical ontology of perdurantism shares more common features with modal realism than the classical ontology of endurantism does. But it is a consequence of this affinity that the corresponding perdurantist notions of coexistence (and of Existence ${ }^{\mathrm{P}}$ () ) are devoid of temporally-laden connotations. There is no sense in which the 1600 part of Descartes becomes older and its view on the rest of the world changes. And there is, at best, only an indirect sense in which this may be true of the entire perduring Descartes. This, of course, is simply more grist for the Asymmetry Thesis (see Section 4), now generalized in accordance with CASH.

Let me now introduce the relativistic versions of the above notions.

6.4. Existence@, coexistence, HPS-mates, and temporal-likeworlds in Minkowski spacetime. According to $\mathrm{CASH}$, coexistence in Minkowski spacetime is a matter of sharing a hyperplane of simultaneity (HPS), as reflected in the statements of CE-CASH, $\mathrm{CE}^{*}$-CASH, and their perdurantist counterparts (Section 6.1). In difference from the classical case, there is no privileged family of HPSs. But the notion of sharing a common HPS is as close as one can get to the classical notion of sharing a moment of common time. In fact, an HPS represents a moment of time in a particular frame of reference. Just like its classical counterpart, it stretches across the entire world. Indeed, the occupant of a given HPS is the world at a certain moment of time in some inertial frame. Let us refer to it as a temporal-like world, and let us call the inhabitants of a temporal-like world HPS-mates. Such inhabitants may be enduring objects wholly present at spacetime points lying on a given HPS, or momentary temporal parts of perduring objects. HPSmates all coexist with each other in an interesting sense. And for any entity wholly present at a spacetime point, there is a sense in which its HPS-mates exist@. But in light of the Asymmetry Thesis, the interesting notion of coexistence and the corresponding notion of Existence@ are temporally-laden only for the endurantist, not for the perdurantist. (More on this in the next two sections.)

For all practical purposes, the relativistic notions based on CASH are indistinguishable from their classical limits when they are applied to objects of everyday life. Thus what has just been said about Descartes and his contemporaries remains valid in the relativistic framework. In general, an inquiry about what enduring objects coexist 
Relativity, Coexistence and Temporal Parts

with what other objects in relativistic spacetime should start with a particular object of interest $\mathrm{E}$ wholly present at spacetime point $\mathrm{O}$ at a certain time $t$ in its life career (i.e., its proper time) and then pose the question as to what objects it coexists with (in the sense of $\mathrm{CE}-\mathrm{CASH}$ ). Other objects enter into such a relation of coexistence with $\mathrm{E}$ at $\mathrm{O}$ by being wholly present at spacetime points sharing a common HPS with O. On this approach, E at $\mathrm{O}$ turns out to coexist (as it should) with what we would pre-relativistically count as its 'contemporaries' and not coexist with any of its 'predecessors'.

The notion of a relativistic 'contemporary' is, of course, different from its classical counterpart. Classical contemporaries exist at the same moment of a single time, the absolute Newtonian time. No such concept is available in the relativistic framework. But there is a good substitute. Each relativistic contemporary of $\mathrm{E}$ at $t$ is a certain age, the age in question being measured by the proper time of that contemporary. In the end, this enables all relativistic contemporaries, including $\mathrm{E}$ itself, to enter into a single many-place relation of coexistence with each other on a par. Thus, the 30-year old Data, the 46-year old Captain James T. Kirk, and the 65-year old Captain Jean Luc Picard are contemporaries of each other; they are HPS-mates. On the other hand, Klingon Trevor is the relativistic predecessor of all of them: none of his momentary locations shares a temporal-like world with any momentary location of the first three (Figure 3).

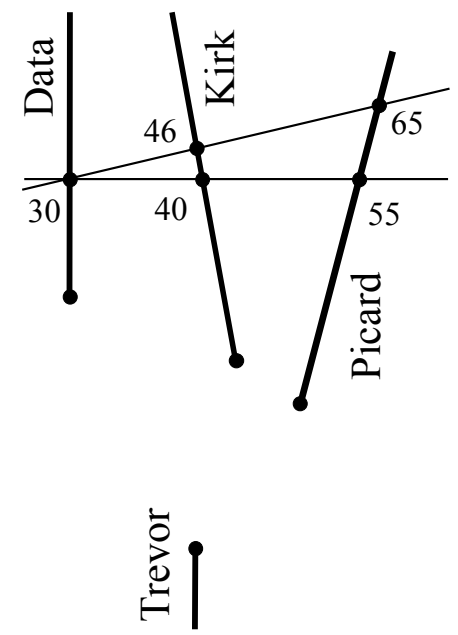

Figure 3.

Notably, the same 30-year old Data is also a contemporary of the 40-year old Captain Kirk and the 55-year old Captain Picard. This is a consequence of the latitude allowed by relativistic spacetime. It may be surprising but it does not look perniciousnot yet. But the point to note here is that, unlike classical temporal worlds, their relativistic descendants may 'crisscross': two HPSs may intersect 'at an angle' in spacetime and thus contain the same enduring object of a certain age or the same stage of a perduring object. This is in addition to the sense in which even classical temporal 
worlds, which do not literally intersect in spacetime (since the absolute HPSs they populate are always parallel), may nonetheless overlap in virtue of containing the same enduring object.

\section{Contextuality}

Let us return, for a moment, to the situation discussed in connection with the Absurdity Thesis. Enduring object $\mathrm{E}$ at $\mathrm{O}$ coexists*, in the sense of $\mathrm{CE}^{*}-\mathrm{CASH}$, with $\mathrm{E}_{1}$ and with $\mathrm{E}_{2}$ but not with both: there is no single temporal-like world containing $\mathrm{O}$ and some momentary locations of both $E_{1}$ and $E_{2}$ (Figure 4). Informed by Gilmore's reasons against the Absurdity Thesis, we may wonder, however, why there should be such a single world, given that $E_{2}$ lies entirely in the absolute future of $E_{1}$ and that, consequently, $\mathrm{E}_{1}$ and $\mathrm{E}_{2}$ fail to coexist with each other. $\mathrm{E}_{1}$ may be Captain Kirk's greatgrandfather and $E_{2}$ his great-grandson. It would be rather strange for anyone to be a 'contemporary' of both.

Figure 4.

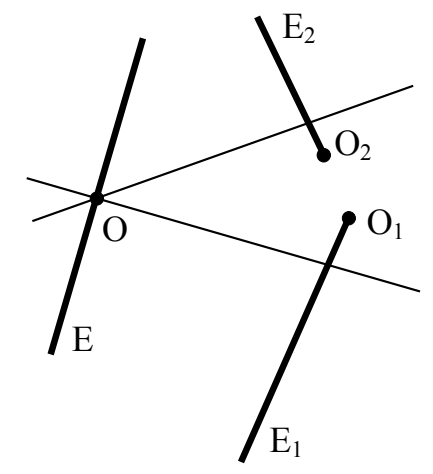

So far so good. But the problem is easily reproduced by separating $E_{1}$ and $E_{2}$ widely enough in space (Figure 5). There we have a situation in which $\mathrm{E}_{1}$ does coexist (in the sense of CE-CASH) with $\mathrm{E}_{2}$ (their momentary locations $\mathrm{O}_{1}$ and $\mathrm{O}_{2}$ share a common HPS), and $\mathrm{E}$ at $\mathrm{O}$ coexists with $\mathrm{E}_{1}$ at $\mathrm{O}_{1}$ and $\mathrm{E}_{2}$ at $\mathrm{O}_{2}$ - but not with both.

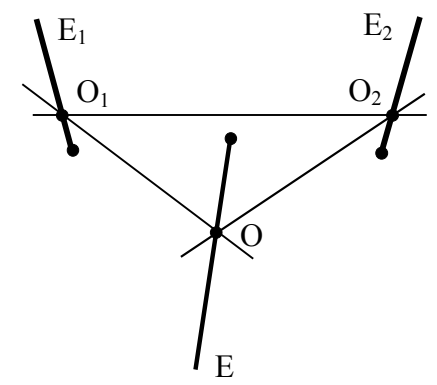

Figure 5. 


\section{Relativity, Coexistence and Temporal Parts}

A similar feature is characteristic of the notion of overlap. This notion is a further generalization of Coexistence* and was briefly mentioned in Section 1.4. The CASH endurantist version of it can be defined as follows:

(E-Overlap) Enduring objects $\mathrm{E}_{1}, \ldots \mathrm{E}_{n}$, considered in abstraction from their locations, temporally E-overlap iff there are spacetime points $\mathrm{O}_{1}$, $\ldots \mathrm{O}_{n}$, such that $\mathrm{E}_{1}, \ldots \mathrm{E}_{n}$ are wholly present at $\mathrm{O}_{1}, \ldots \mathrm{O}_{n}$ respectively and $\mathrm{O}_{1}, \ldots \mathrm{O}_{n}$ belong to a common HPS.

Consider three enduring objects that come to be and cease to exist. Then they may coexist pairwise at some point or other of their life careers. In other words, they may Eoverlap. But this does not guarantee that they coexist (in the same sense) all together. The failure of such mutual coexistence occurs in cases in which no selection of momentary locations of the three objects can be unified by a single triadic HPS-mate relation. Thus it may be the case that Data E-overlaps with Captain Kirk, Captain Kirk E-overlaps with Captain Picard, and the latter with Data. Taken pairwise, they all share temporal-like worlds with each other. Taken all together, however, they do not share any single temporal-like world (Figure 6).

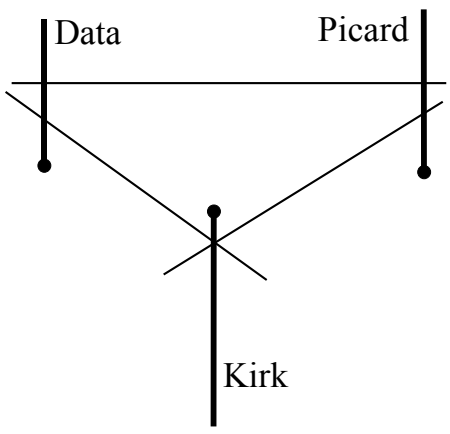

Figure 6.

The same is true of a quadruple of objects. All the triples constructed out of them may coexist, in the sense of CE-CASH or E-overlap; but this does not entail the coexistence or E--overlap of all four. (Considerations of space prevent me from illustrating such a case.)

These results, which can be extended to more numerous collections of objects, show that coexistence in the relativistic world of enduring objects is partitioned in a most peculiar way bearing the mark of contextuality: facts about coexistence of the members of a collection of objects, however numerous, become sensitive to what other objects are taken into account. Such facts do not 'add up' properly.

Contextuality of coexistence should not be confused with the breakdown of transitivity. As we know by now, the various relations of coexistence between members 
of pairs of objects in the relativistic world generally fail to be transitive. For example, coexistence, in the sense of E-Overlap, of $E$ with $E_{1}$ and of $E$ with $E_{2}$ does not entail the coexistence, in the same sense, of $\mathrm{E}_{1}$ with $\mathrm{E}_{2}$. Contextuality, on the other hand, means that the coexistence between the members of all such pairs does not entail the coexistence among the members of the whole triple: $\mathrm{E}, \mathrm{E}_{1}$, and $\mathrm{E}_{2}$.

Why should anyone be bothered by contextuality but not so much by the lack of transitivity? One reason is that transitivity fails, for some senses of coexistence, even in the classical situation. I coexist (i.e., temporal overlap) with my father and he coexists (in the same sense) with my grandfather. But I do not coexist with my grandfather. But the classical relation of coexistence is free of contextuality. If my father, my grandfather, and I overlap pairwise then we overlap all together. We do not find this remarkable because we simply take it for granted. But for the endurantist who takes relativity seriously, something important is at stake here.

Indeed, enduring objects live in temporal-like worlds; those are where they are wholly present and those are what they share - much as objects in Lewis's ontology live in and share particular possible worlds. In a trivial sense, the latter also share the entire collection of worlds. But nothing significant turns on this broader notion. All the important features of the possible worlds ontology, including the modal properties of objects, are grounded in the facts about what objects belong to what worlds. The fact that every object also belongs to the whole collection of worlds bakes no bread. Similarly, the endurantist should take the facts about what object belongs to what temporal-like world at what point in its career - and what other objects it shares that world with - as the ground of all the important features exhibited in the temporal 'multi-universe'. These include temporary properties of objects and their changing relations with each other. The fact that all enduring objects trivially share the single spacetime manifold gives no further purchase.

The endurantist must thus recognize existence in a temporal (or temporal-like) world and sharing such a world as basic facts. But then it is natural to expect such facts to obey a reasonable 'calculus'. And they certainly do so in the classical case, where the $t$-mate relation is grounded in the absolute simultaneity among momentary locations of enduring objects. Minkowski spacetime suggests a bona fide candidate to do a similar job, the HPS-mate relation, which adequately recovers its pre-relativistic counterpart in the classical limit. The problem with it is that it stumbles upon a simple rule that is, intuitively, part and parcel of the concept of coexistence: to put it in a grotesque form, if any 999 members of a collection of 1000 objects coexist with each other, then all 1000 objects must do so as well.

But isn't the same true of momentary parts, or stages, of perduring objects? After all, aren't they similar to enduring objects in this respect at least that they are wholly present at spacetime points? If so, the stages' coexistence relations with each other in the relativistic world must be partitioned in the same way as the corresponding relations of 


\section{Relativity, Coexistence and Temporal Parts}

enduring objects taken at certain moments of their careers. And if the latter fail to obey a 'reasonable calculus', then so must the former.

I cannot but agree with this. And I take this to be a reason to prefer the perdurance theory of persistence to the stage theory (see note 4 on the stage theory). The argument to this effect is developed elsewhere (Balashov, 2002). Our target here, however, is endurantism, not the stage theory. And it has already emerged in our discussion of the Asymmetry Thesis that perduring objects do not 'live' in temporal-like worlds: they are too long to fit in there. The fact that their momentary parts do provides an indirect sense in which one could speak of the coexistence relations among perduring wholes 'taken' at certain moments of their individual times. But such relations are of secondary importance to the perdurantist ontology. Their failure to obey a 'reasonable calculus' is, therefore, metaphysically inconsequential.

I submit that the best way to categorize such derivative relations is to treat them as perspectival relations in spacetime. A spatial analogy may be helpful here. A typical art museum has numerous exposition rooms variously connected to each other by wide entrances on their sides. There may be a location in one of the rooms from which one can see through several such openings and thus 'line up' several rooms in a single line of sight. One can even manage to see some paintings or parts thereof in distant rooms. Slightly changing one's location may significantly affect the arrangement of the rooms in the line of sight: some openings may disappear from it and others emerge. There may be a perspective in which Room 101, say, is lined up in this way with Rooms 102, and 104; another perspective in which Rooms 101, 103 and 104 are so lined up; and another in which Rooms 102, 103, and 104 are. But there may be no single perspective in which all four rooms could be lined up. This tells us something informative about the floor plan of the museum. But it has no further implications.

Similarly, the distribution of perduring objects in spacetime tells us something useful about the way their stages relate to each other. But given the central thesis of perdurantism - that ordinary objects are four-dimensional entities and not stages ${ }^{8}$-we should view the spatio-temporal relations among the stages as merely perspectival features of a collection of perduring wholes, exhibited in the single spacetime manifold, not as fundamental facts about their existence and coexistence. Unlike enduring objects (and unlike stages), such wholes do not live in (and hence, cannot share) temporal-like worlds. What counts as a world for the former is no more than a perspective for the latter.

Contextuality is only part of the price endurantism has to pay in the transition to relativity. Besides being contextual, coexistence in the relativistic world of enduring objects may exhibit chronological incoherence.

\footnotetext{
${ }^{8}$ See the end of Section 4 for the importance as well as the counterintuitive nature of this thesis, and for its role in dissolving the problem of coincident objects.
} 


\section{Chronological Incoherence}

Let us start with an unproblematic case. The 30-year old Data coexists with the 35-year old Captain Kirk and the 40-year old Captain Picard: they share a common temporal-like world occupying $\mathrm{HPS}_{1}$. As Data grows older and reaches the age of 35, he happens to coexist with the younger Captain Kirk and Captain Picard, who both have just turned 32: the three characters share a common temporal-like world occupying $\mathrm{HPS}_{2}$ (Figure 7).

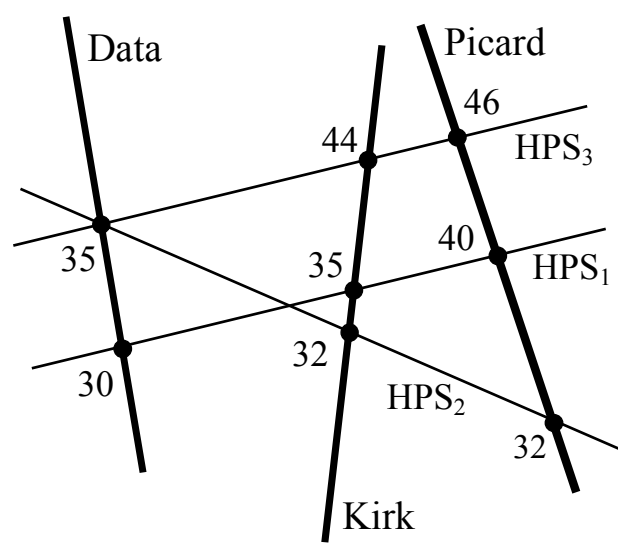

Figure 7.

This, however, should not be viewed as being particularly disturbing. At any moment in his life, Data belongs to an infinite number of temporal-like worlds. The fact that one can pick out a chronologically incoherent series of such worlds (e.g., those occupying $\mathrm{HPS}_{1}$ and $\mathrm{HPS}_{2}$, in this order) should not be held against the endurantist, as long as another, chronologically coherent series is available; for example, the series including $\mathrm{HPS}_{1}$ and $\mathrm{HPS}_{3}$. The temporal-like world occupying $\mathrm{HPS}_{3}$ features the 35-year old Data, and the correspondingly older Captain Kirk and Captain Picard. One is not saddled with the offending sequence of $\mathrm{HPS}_{1}$ and $\mathrm{HPS}_{2}$, because there is no reason to allow one to exploit the latitude inherent in relativistic spacetime frivolously, by sequencing temporal-like worlds at will. The availability of chronologically coherent series of such worlds is all that the StarTrek biographer needs to tell a sensible story about the life careers of the three famous characters and their relations to each other.

But there are cases where a chronologically coherent series of temporal-like worlds is not available (unless one makes such a series improperly short) and there is no escape from a disturbing conclusion that ageing results in being a contemporary of progressively younger companions. One case of this sort is sketched in Figure 8. Here the most one can do is to identify a chronologically coherent series of temporal-like 
worlds containing the correspondingly ageing Data, Kirk, and Picard, for example, some series including $\mathrm{HPS}_{1}$ and $\mathrm{HPS}_{2}$, in this order. Adding Trevor to the picture, however, turns the series into a bad one. As Data, Kirk, and Picard all grow older, they find themselves in worlds with the younger and younger Trevor. And that is not the worst possible scenario yet. With some modifications, one could make progressively ageing Data, Kirk, and Picard unavoidable contemporaries of, first, Chief Trevor, next the 15year old Cadet Trevor, then the newly born Klingon baby just named Trevor, and eventually, Trevor's great-grand-grandfather! This is surely an unwelcome result. Along with contextuality, it brings out the difficulties of formulating the many-place coexistence relations in the relativistic world of enduring objects.

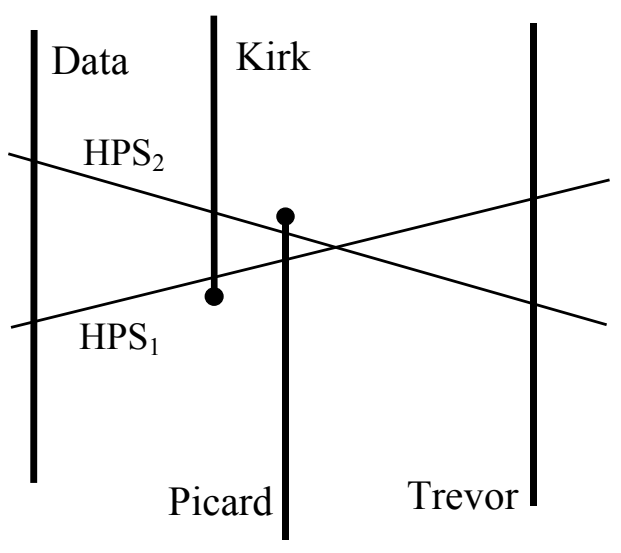

Figure 8.

Why is perdurantism not afflicted with the same or similar problems? One reason has been noted earlier: there is no strict sense in which perduring objects can be said to age. The bearing of considerations having to do with ageing on perdurantism is, therefore, indirect and ontological harmless. This is sufficient to uphold the asymmetry between perdurantism and endurantism in the face of chronologically incoherent series.

But it is worth repeating that there is another and more general aspect of the asymmetry that pertains both to contextuality and chronological incoherence, even though the former is a 'static' phenomenon, having to do with a collection of objects taken at particular moments of their individual times or in complete abstractions from them, whereas the latter is a 'dynamic' phenomenon having to do with ageing. The feature common to both of them is that perduring objects do not occupy the sort of habitats (i.e., temporal-like worlds confined to single HPSs) that may exhibit contextuality or chronological incoherence of coexistence. All perduring entities coexist with each other in the Minkowski world. This does not mean that one cannot pose temporally qualified questions about the coexistence of their various parts. And the 
answers to them are informative as they tell one something about the overall distribution of perduring objects in spacetime. But the CASH value of such information is perspectival, not ontological.

\section{Another Objection}

Contextuality and chronological incoherence of multigrade coexistence relations in Minkowski spacetime could, in principle, be mitigated by identifying the loci of temporal-like worlds, not with flat hyperplanes of simultaneity, as CASH prescribes, but with generic spacelike hypersurfaces of variable curvature. ${ }^{9}$ Adopting this course would preclude the offending situations described in Sections 7 and 8 from arising, because the following mathematical fact about Minkowski spacetime obtains:

(FACT) For any set of pairwise spacelike separated points $O_{1}, \ldots O_{n}$ in Minkowski spacetime, there is a continuous three-dimensional spacelike surface containing $O_{1}, \ldots O_{n}$.

FACT is non-trivial, and the proof, on behalf of the objector, is given in the Appendix. ${ }^{10}$

But I think doubts can be raised as to whether curved spacelike hypersurfaces would provide an adequate framework for temporal-like worlds in Minkowski spacetime. First, the spatial geometry of such worlds would, in general, be non-Euclidean. Second, the non-trivial nature of FACT (see note 10) and the complicated profile of function $F$ (see Appendix) strongly suggest (even if they do not strictly establish) that getting around contextuality and chronological incoherence in sufficiently complex situations would result in exceedingly 'bent' and 'unstable' temporal-like worlds - unstable in the sense that small shifts in the mutual arrangement of objects and adding new objects to existing collections could induce drastic disturbances in the profile of the resulting 'coexistence surface' and its spatial geometry. Note that disturbances of this sort may occur in locations far away from the shifts precipitating them (another manifestation of contextuality or, perhaps, 'non-locality'?).

For example, as regards contextuality, suppose three objects coexisting in a certain spatially flat temporal-like world form an Euclidean triangle there (i.e., a triangle

\footnotetext{
${ }^{9}$ I thank Cody Gilmore and the referee for this Journal for impressing upon me the importance of addressing this objection.

${ }^{10}$ To see that FACT is indeed non-trivial, note that a hyperplane defined by a triangle of three pairwise spacelike-related points in $2+1$ spacetime need not be everywhere spacelike. Assume $c=1$ and the following Cartesian coordinates of four points: $\mathrm{O}_{1}(-10,0,0), \mathrm{O}_{2}(0,-10,11), \mathrm{O}_{3}(0,10,11)$, and $\mathrm{O}$ $(0,0,11)$. While $\mathrm{O}_{1}, \mathrm{O}_{2}$ and $\mathrm{O}_{3}$ are pairwise spacelike separated, $\mathrm{O}_{1}$ and $\mathrm{O}$ (which is the midpoint of the line segment $\mathrm{O}_{2} \mathrm{O}_{3}$ ) are not.
} 
whose angles sum up to two right angles). Adding other objects to the coexistence pool could make things go 'wild', even among the members of the original group: the triangle they define might suddenly stop being Euclidean, and this for no physical reason.

Similarly, a 'temporal' sequence of curved surfaces that preserves chronological coherence may feature members so peculiarly bent that doubts may arise that saving coherence at such a cost would be a good deal. For example, a series of temporal-like worlds preserving chronological coherence in the situation depicted in Figure 8 would feature significantly ageing Data, Kirk, and Trevor (say, by decades) along with Picard who would be ageing only minutely, say, by a fraction of a year-or a month, or a second! Out of the frying pan and into the fire?

These observations suggest, then, that irregularly curved spacelike surfaces do not, in general, provide natural and well-behaved receptacles for temporal-like worlds. One might think that a better candidate for this role would be a uniform family of hypersurfaces associated with cosmological time in smoothened-out models of the universe. But since such a series would lack any 'flexibility' (even the flexibility enjoyed by HPSs stretching through spacetime at arbitrary 'angles'), it would only exacerbate the original problems. ${ }^{11}$

\section{Conclusions}

The foregoing discussion prompted by Gilmore's enlightening critique has brought forth some important lessons. First, the interesting notion of coexistence has genuinely ontological significance for endurantism, but not for perdurantism. This serves to uphold the Asymmetry Thesis (more precisely, its CASH-based modification). Second, the interesting notion of coexistence must be grounded in a multigrade relation among the momentary locations of enduring objects in Minkowski spacetime (or of momentary stages of perduring objects). Accordingly, CASS must be replaced with CASH, which is, arguably, the best account of (the interesting notion of) coexistence in the relativistic context. In light of this modification, it becomes clear that the original version of the Absurdity Thesis, focused on the temporal connotations of the 'togetherness claim', cannot be sustained. This sets Gilmore's objection to it in perspective. The objection must be conceded by anyone committed to CASS. But the replacement of CASS with $\mathrm{CASH}$ also suggests that the simple togetherness claim is only one, and relatively

${ }^{11}$ The brief discussion above suggests that the issues raised in the series of papers exploring the impact of SR for the metaphysics of persistence (see note 1) must eventually be extended to the general relativistic context. Although Minkowski spacetime is a good approximation of most of the spacetime of our world, it is, in the end, just that: an approximation. Still, metaphysical implications of such an approximation are valuable, and there is a long tradition of debating them; e.g., the Putnam-Stein debate continuing to the present day. 
innocent, instance of a more general problem afflicting the many-place relation of coexistence in the relativistic world of enduring objects. Two more harmful manifestations of this problem are contextuality and chronological incoherence. They strike at the very heart of the endurantist ontology, the notion of being wholly present at a single moment of time (in a given reference frame). The perdurantist, on the other hand, is free to interpret the peculiar consequences of the coexistence relation in the Minkowski world as merely perspectival features of its intrinsic geometry. I conclude that perdurantism remains a preferred theory of persistence in the relativistic context. ${ }^{12}$

\section{Appendix: Proof of FACT}

Consider a set of points $\mathrm{O}_{1}, \ldots \mathrm{O}_{n}$ in Minkowski spacetime. For all $i \neq j, \mathrm{O}_{i}$ and $\mathrm{O}_{j}$ are spacelike separated. Prove that there is a continuous spacelike $3 \mathrm{D}$ surface ${ }^{13}$ containing $\mathrm{O}_{1}, \ldots \mathrm{O}_{n}$.

Def.: A function $f$ on a metric space $X$ is $K$-Lipschitzian just in case $\exists K>0$ such that for all $x, x^{\prime} \in X,\left|f(x)-f\left(x^{\prime}\right)\right| \leq K d\left(x, x^{\prime}\right)$.

Lemma: If functions $f_{1}, \ldots f_{n}$ on $X$ are $K$-Lipschitzian, then their lower bound $f \equiv \inf _{i=1 \ldots n} f_{i}$ is $K$-Lipschitzian.

Proof of Lemma, by induction:

1. Base step. Assume $f_{1}$ and $f_{2}$ are $K$-Lipschitzian. Fix two points $x$ and $y$. Let $g$ $\equiv \inf \left(f_{1}, f_{2}\right)$. We have:

$$
\begin{aligned}
& -K d(x, y) \leq f_{1}(x)-f_{1}(y) \leq K d(x, y) \\
& -K d(x, y) \leq f_{2}(x)-f_{2}(y) \leq K d(x, y)
\end{aligned}
$$

Suppose $f_{1}(x) \leq f_{2}(x)$. (Otherwise exchange $f_{1}$ and $f_{2}$.) Then $g(x)=f_{1}(x)$. Consider two cases: (a) $f_{1}(y) \leq f_{2}(y)$ and (b) $f_{1}(y)>f_{2}(y)$.

(a) If $f_{1}(y) \leq f_{2}(y)$ then $g(y)=f_{1}(y)$ and, from (1): $-K d(x, y) \leq g(x)-g(y) \leq K d(x, y)$.

(b) If $f_{1}(y)>f_{2}(y)$ then $g(y)=f_{2}(y)$. From (1): $g(y)=f_{2}(y)<f_{1}(y) \leq f_{1}(x)+K d(x, y)$ $=g(x)+K d(x, y)$.

Therefore, $g(y)-g(x)<K d(x, y)$.

\footnotetext{
${ }^{12}$ I am indebted to Cody Gilmore and Dean Zimmerman for comments on earlier drafts. Special thanks are due to a referee for this Journal for very constructive criticism and many helpful suggestions. Finally, I thank my son Alex Balashov for a crash course on StarTrek.

${ }^{13}$ For our purposes, a surface is spacelike just in case any two points on it are spacelike separated; we do not require differentiability. But we shall accept, without proof, that if a sought-for continuous, but perhaps 'corrugated', surface is available, it can always be appropriately 'smoothened' and be made everywhere differentiable, thus becoming, in a technical sense, a hypersurface.
} 
From (2): $g(y)=f_{2}(y) \geq f_{2}(x)-K d(x, y) \geq f_{1}(x)-K d(x, y)=g(x)-K d(x, y)$.

Therefore, $g(y)-g(x) \geq-K d(x, y)$.

2. Inductive step. Assume: If functions $f_{1}, \ldots f_{n}$ on $X$ are $K$-Lipschitzian, then $f \equiv \inf _{i=1 \ldots n} f_{i}$ is $K$-Lipschitzian. Show: If functions $f_{1}, \ldots f_{n+1}$ on $X$ are $K$-Lipschitzian, then $f \equiv \inf _{i=1 \ldots n+1} f_{i}$ is $K$-Lipschitzian.

Denote: $g \equiv \inf _{i=1 \ldots n} f_{i} . \quad$ By assumption, $g$ is $K$-Lipschitzian. Note that $\inf _{i=1 \ldots n+1} f_{i}=\inf \left(g, f_{n+1}\right)$. Apply the result of the base step to $g$ and $f_{n+1}$.

Main proof. Pick a frame. Set $c=1$. We are looking for a continuous real function $F$ on $X$ satisfying $|F(x)-F(y)|<d(x, y)$ for all distinct $x$ and $y$ and such that $F\left(x_{i}\right)=t_{i}, i=1, \ldots n$.

Let $K=\min _{\substack{1 \leq i \leq n \\ 1 \leq j \leq n \\ i \neq j}} \frac{\left|t_{i}-t_{j}\right|}{d\left(x_{i}, x_{j}\right)}$ and $F_{i}(x) \equiv t_{i}+K d\left(x, x_{i}\right)$. Obviously $K<1$. Define $F \equiv \inf _{i=1 \ldots n} F_{i}$

By the triangle inequality, $\left|F_{i}(x)-F_{i}(y)\right|=\left|K d\left(x, x_{i}\right)-K d\left(y, y_{i}\right)\right| \leq K d(x, y)$. Therefore all $F_{i}(x)$ are $K$-Lipschitzian. By Lemma, $F(x)$ is $K$-Lipschitzian. Hence $F(x)$ is continuous and $|F(x)-F(y)|<K d(x, y)$. Since $K<1$, this entails, for all distinct $x$ and $y$, $|F(x)-F(y)|<d(x, y)$.

It remains to show that $F\left(x_{i}\right)=t_{i}, i=1, \ldots n$. First, note that $F_{i}\left(x_{i}\right)=t_{i}$. Next, for all $j \neq i, F_{j}\left(x_{i}\right) \geq t_{i}$. Hence $F\left(x_{i}\right) \equiv \inf _{j=1 \ldots n} F_{j}\left(x_{i}\right)=t_{i} \cdot{ }^{14}$

${ }^{14}$ The proof strategy and the choice of $F$ are due to Tom Goodwillie. I was also greatly helped by Valery Alexeev who patiently educated me on Lipschitzian functions. I am grateful to both of them. 
Yuri Balashov

\section{References}

Balashov, Y. (1999): 'Relativistic Objects', Noûs 33, 644-662.

- (2000a): 'Enduring and Perduring Objects in Minkowski Space-Time', Philosophical Studies 99, 129-166.

(2000b): 'Relativity and Persistence', Philosophy of Science 67 (Proceedings), S549-S562.

(2000c): 'Persistence and Space-Time: Philosophical Lessons of the Pole and Barn', The Monist 83, 321-340.

(2002): 'On Stages, Worms, and Relativity', in Craig Callender (ed.), Time, Reality, and Experience (pp. 223-252), Cambridge: Cambridge University Press.

- (2003): 'Temporal Parts and Superluminal Motion', Philosophical Papers 32, 113.

Gilmore, C. (2002): 'Balashov on Special Relativity, Coexistence, and Temporal Parts', Philosophical Studies 109, 241-63.

Hales, S.D. and Johnson, T. (2003): 'Endurantism, Perdurantism, and Special Relativity', The Philosophical Quarterly 213, 524-539.

Haslanger, S. (2003): 'Persistence Through Time', in M.J. Loux and D. Zimmerman (eds.), The Oxford Handbook of Metaphysics (pp. 315-354), Oxford: Oxford University Press.

Hawley, K. (2001): How Things Persist, Oxford: Clarendon Press.

Heller, M. (2000): 'Temporal Coincidence is not Overlap', The Monist 83, 362-380.

Hudson, H. (2002): 'Moving Faster than Light', Analysis 62, 203-205.

- (2003): 'Immanent Causality and Diachronic Composition: A Reply to Balashov', Philosophical Papers 32, 15-23.

Lewis, D. (1986): On the Plurality of Worlds, Oxford: Blackwell.

Rea, M. (1998): 'Temporal Parts Unmotivated', The Philosophical Review 107, 225-260.

Sider, T. (2001): Four-Dimensionalism: An Ontology of Persistence and Time, Oxford: Clarendon Press. 